\title{
Design and Evaluation of SIMpliLife, an NFC Platform to Ease Day-to-Day Actions of People's Life
}

\author{
Carlo Maria Medaglia, Alice Moroni, Serena Sposato, Ugo Biader Ceipidor and Ylenia De Rosa \\ CATTID, Sapienza University of Rome, Rome 00185, Italy
}

Received: April 20, 2011 / Accepted: May 20, 2011 / Published: June 25, 2011.

\begin{abstract}
In this paper the authors describe the design, prototyping and evaluation of SIMpliLife, a framework for mobile phones with the aim of making people's life easier, providing day-to-day services such as payment, ticketing and information retrieval by means of NFC (Near Field Communication) technology. It was developed via the collaboration between two research labs of Sapienza University of Rome: the RFID (Radio Frequency Identification) Lab and the Usability and Accessibility Lab (LUA), both belonging to CATTID (Centre for Applications of Teleservices and of Technologies for Innovation in Digital world) research centre. Technically, SIMpliLife is a SIM-based platform for NFC mobile phones that interfaces several applications able to manage the abovementioned services. The high-level User Interface has been developed by means of Smart Card Web Server (SCWS) technology, thus providing enhanced user experience if compared to traditional SIM-based implementations based on SIM Toolkit.
\end{abstract}

Key words: Near field communication (NFC), smart card web server (SCWS), user-centered design (UCD), universal integrated circuit card (UICC).

\section{Introduction}

In the last couple of decades, the miniaturization of electronic components inside consumer devices, as well as the growing availability of wireless technologies capable of connecting smart objects to the Internet, have somehow realized an Ubiquitous Computing environment, as predicted by Mark Weiser in the early 1990s [1-2]. In his works, the researcher from XEROX PARC hypothesized a world of interconnected objects by means of Information and Communication Technologies (ICT), able to change people's daily lives.

Carlo Maria Medaglia, professor, research fields: RFID, HCI, satellite remote sensing and mobile/wireless communication.

Serena Sposato, postgraduate scholar, research fields: NFC, mobile, smart card technology, usability.

Ugo Biader Ceipidor, full professor, research fields: RFID, telecommunications, simulation for learning by doing, networking software, analytical chemistry.

Ylenia De Rosa, postgraduate scholar, research fields: NFC, mobile, SIM application development.

Corresponding author: Alice Moroni, Ph.D. student, research fields: NFC, mobile, user-centered design. E-mail: moroni@cattid.uniroma1.it.
During the same years, another significant process in the ICT field has been progressively completed: the convergence of a number of communication technologies inside one single device, the mobile phone, which, according to the International Telecommunication Union (ITU), is nowadays spread among $97 \%$ of the population of developed economies [3]. The convergence of several communication interfaces (Wi-Fi, Bluetooth, W-CDMA (Wideband Code Division Multiple Access), which is the standard data interface used by the UMTS (Universal Mobile Telecommunications System), as well as RFID (Radio Frequency Identification) in the forming of NFC (Near Field Communication)) inside the mobile phone, makes this device the most suitable in order to access different types of services within an interconnected environment such as the one predicted by Mark Weiser.

Conscious about this scenario, the RFID Lab of CATTID (Centre for Applications of Teleservices and of Technologies for Innovation in Digital world) has 
designed and developed a platform for NFC mobile phones, named SIMpliLife. As the name suggests, it is a SIM-based platform thought to ease day-to-day actions of people's life. It is composed of three separate applications-SIMpliCity, SIMpliPay and SIMpliTravel loaded into the SIM card and linked between each other.

SIMpliLife relies on previous work [4-5] performed by CATTID in designing and developing NFC-based services. In fact, the lab had already developed an application named SIMpliCity [4] and also a ticketing system, named NFCTicketing [5], but these were isolated applications and needed to be separately loaded into the mobile phone. Conversely, SIMpliLife integrates the functions of SIMpliCity and NFCTicketing, giving the user a complete user experience, moreover adding a payment application (SIMpliPay) to the user's portfolio. So, SIMpliLife exploits the real potentiality of NFC technology integrating a set of applications into a single device.

Moreover, SIMpliCity and NFCTicketing were designed for an NFC mobile phone with the Secure Element (SE) embedded in the handset (Nokia 6131 and Nokia 6212), while SIMpliLife was realized for an NFC mobile phone with a SIM-based SE (Sagem my700x), making use of Smart Card Web Server (SCWS) technology with the aim of providing a high-level User Interface (UI). The differences between these approaches will be highlighted in section 2 .

In order to build a fully usable service portfolio, the development team has involved active users in every phase of the process, following the User-Centered Design (UCD) methodology that will be fully described in section 4 .

The paper is organized as follows: Section 2 gives an overview of the NFC technology, while section 3 describes all the architectural components of SIMpliLife, detailing both software and hardware features and the relative communication scheme. In section 4 the attention is focused on the design process which, according to the User-Centered Design methodology, has involved final users at each stage of the implementation. Extensive attention will be given to the usability studies performed on the platform, itemizing the results of the preliminary studies and also the responses, opinions and expectations of the users which we conducted the test with. Finally, section 5 summarizes the conclusion of the study and reports the future work that needs to be done to reach the final release of SIMpliLife.

\section{NFC Technology Background}

NFC is a standardized technology [6-7] that allows data exchange between passive and active devices through a wireless communication working at the frequency of $13.56 \mathrm{MHz}$, with a throughput of 424 $\mathrm{kbit} / \mathrm{s}$, at the maximum distance of $10 \mathrm{~cm}$. NFC devices are provided with an RF antenna, an NFC controller that manages the information retrieved by the antenna and a cryptography chip (a smart card), named Secure Element, that enables the phone to perform secure data exchange such as the one needed for payment transactions (and, in general, for all the authentication processes).

\subsection{Secure Element and Application Architecture}

Although there could be, at least, four alternatives regarding the Secure Element's position [8-9], in this paper we only consider the two which are the most used in the NFC implementation: SE provided by the UICC (Universal Integrated Circuit Card), commonly referred to as "SIM card", and SE as an embedded chip inside the phone, from which the names "SIM-based" and "handset-based" related to the applications developed for an NFC device. Handset-based applications and SIM-based ones are similar but not the same: they share the simple use, typical of the NFC technology, but they work in different ways.

With respect to handset-based applications, the Java Platform Micro Edition (JME) provides a flexible and optimized environment that consists of a set of libraries 
designed according to Sun's philosophy "Write once Run Everywhere". The advantages in using the JME platform are: a highly customizable user interface, the possibility of using local applications also working in off-line mode, high data processing capability, the availability of a generous amount of documentation and the possibility to easily interface with web platforms. On the other hand, the lifecycle of the applications is not so high, in fact, changing the phone involves the unavailability of the existing applications, and also, the portability is not guaranteed, due to the fact that differences between handset devices (operating systems, display resolution, etc.) can lead to subtle differences in how the application may execute.

Conversely, SIM-based applications can rely on the following advantages:

- Security: Currently, UICC satisfies the highest security standards for data management and storage;

- Portability: Applications run on every enabled device;

- Standardization: UICC solution is actually attaining to the international specifications ETSI-SCP [10-11] and Global Platform [12];

- Remote management: Applications can be real-time managed by Over-the-Air (OTA) communication;

- High lifecycle: User can change device without modify the applications loaded into the UICC;

- Universal form factor: The SIM card has a universal form and so all mobile phones can support it.

In order to develop SIM-based applications, two different approaches will be considered: SIM Application Toolkit (SAT) and Smart Card Web Server (SCWS). According to Gemalto, one of the major companies involved in smart card and SIM card development, the SIM Application Toolkit (SAT) is a set of commands which define how the card should interact with the outside world and extends the communication protocol between the card and the handset. With SAT, the card has a proactive role with the handset (this means that the SIM initiates commands independently of the handset and the network) [13]. The advantages in using SAT are the high level of interoperability between different mobile devices, high levels of security through identity verification and encryption, the deploy on the largest number of mobile devices. Nevertheless, the user interface is only text-based so the user experience is not so satisfactory.

Conversely, SCWS is an emerging technology that allows to build interactive services running on the UICC with a highly customizable User Interface. SCWS inherits the abovementioned advantages of the SIM-based approach (security, portability, standardization, remote management, high life-cycle and universal form factor), adding a more satisfactory user experience. From the technical point-of-view, the SCWS is a Web server running on the UICC [14]. It provides static html pages, images and multimedia content, allowing to dynamically manage external html pages via the mobile phone's browser. SCWS differs from Java implementations by using standard Internet technologies such as html and http/https.

\subsection{Secure Element and Mobile NFC Ecosystem}

Two important actors of the mobile NFC ecosystem respectively support handset-based and SIM-based approaches: the mobile phone manufacturers (together with banks and other service providers) and the Mobile Network Operators (MNOs). MNOs which are interested in NFC would like to see the applications stored on the Subscriber Identity Module (SIM) card which they issue and not on a separate tamper-resistant chip that may come embedded in many NFC phones. Conversely, banks and other service providers would prefer to manage a SE which is not under MNO's control [15].

The GSM Association (GSMA), a global association representing more than 700 MNOs across 218 countries in the world, supports the SIM-based solution. In 2007 the GSMA published a technical guideline white paper on NFC services, providing the MNOs 
views on them [16]. Moreover, the GSMA has launched two initiatives to support the SIM-based approach for NFC application development: the Mobile NFC initiative, supported by nineteen MNOs, which have worked together to develop a common vision on Mobile NFC services, promoting the development of a stable and efficient ecosystem and to prevent market fragmentation [17] and the Pay-Buy-Mobile project, supported by thirty-four of the world's largest MNOs [18].

The SIM-based solution seems to be the best not only from a technical point-of-view, but it is also preferred by a wide and strong set of actors in the mobile NFC ecosystem. That's why we have chosen this solution for the development of SIMpliLife.

\section{SIMpliLife Solution}

As abovementioned, SIMpliLife is a SIM-based platform for NFC mobile phones able to interface several applications and manage many different services. In the first prototype we integrated three different applications to cover the most popular NFC use cases: information retrieval via RFID passive tags (SIMpliCity), payment (SIMpliPay) and ticketing (SIMpliTravel), but it would be possible to add new applications following the same architectural principles.

More in detail, SIMpliCity has been designed to help tourists or local citizens while sightseeing around the city. By placing Smart Posters provided with RFID passive tags in train stations, airports, tourists offices etc., SIMpliCity is able to give information about selected points-of-interest of the "City", "SIMply" tapping the NFC mobile phone near the highlighted area of the Smart Poster (see Fig. 1).

Once the phone has been tapped near the RFID tag, the user can access several services such as knowing how to reach the city's highlighted places using public transportation system or obtaining information about points-of-interest and entertainment. Moreover, thanks to SIMpliCity, users are able to call a taxi in an automatic way, overcoming the difficulty of the foreigner tourists

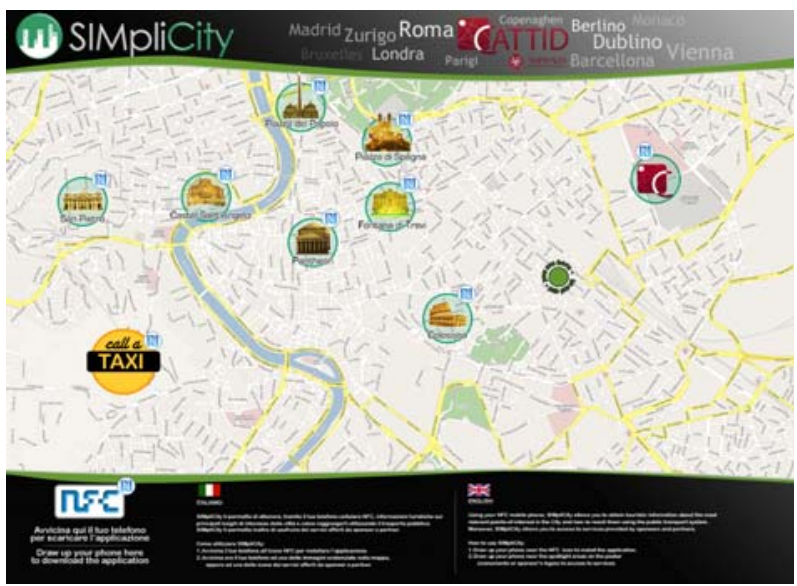

Fig. 1 SIMpliCity smart poster.

that might not be able to explain where they are as they have just arrived in a city where they have never been before.

Thanks to the integration with SIMpliPay, users are not only able to request information about the points-of-interest, but they can also buy entrance tickets with just one more click. In fact, if the user selects the "tourist information" button, he can then choose the "buy ticket" option and he will be automatically redirected to SIMpliPay for the selection and payment of the entrance ticket (see Fig. 2).

In this case the user is performing a remote payment by means of an OTA connection with PayPal server. We have chosen a PayPal-based system, as it is already familiar to users and because PayPal offers the necessary APIs to integrate its check-out method on mobile websites. By a remote payment, the user can buy entrance tickets for the monuments highlighted in SIMpliCity Smart Poster, or buy tickets for public transportation: in this case the ticket selection is managed by SIMpliTravel and will be detailed later.

Using SIMpliPay the user is able to perform also proximity payments, exploiting the real potential of NFC technology. To start a proximity payment the user will select "NFC Payment" on the main menu of SIMpliPay: This action recalls a Servlet that waits until the user taps his mobile phone near the reader (on which the payment amount is loaded). By the user's "drawing up" action, the payment is performed and the 


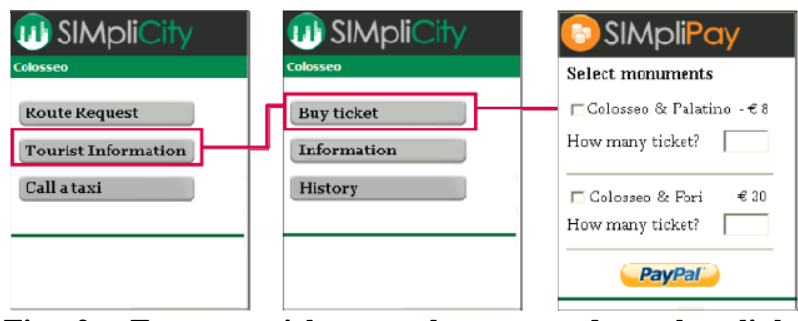

Fig. 2 Entrance ticket purchase procedure that links SIMpliCity and SIMpliPay.

payment amount is decreased from a mobile wallet pre-loaded into a protected area of the SIM card, which acts as a Secure Element.

Finally, SIMpliTravel allows the user to buy and validate tickets for public transportation. The user can select to pay Over-The-Air using his PayPal account, or to perform a proximity payment via NFC technology. Independently from the payment method chosen, tickets will be charged into the SIM-based Secure Element. To validate one or more tickets, the user will select the "Validate tickets" button on SIMpliTravel main menu, then he will select how many tickets he wants to validate and finally he will draw up the phone near the NFC validating machine.

From a technical point-of-view, SIMpliLife is composed by the following HW and SW components:

- An NFC mobile phone (Sagem my700x);

- A Smart Poster provided with RFID passive tags;

- A SIM card provided with SCWS and NFC technologies;

- SIMpliCity Servlet enabled to read tags and to dynamically realize web pages based on the tag content;

- SIMpliPay Servlet enabled to communicate using the NFC protocol, to open http/https connections with a remote server (PayPal), and also to manage (check and top up) a mobile wallet loaded in the SIM card;

- SIMpliTravel Servlet enabled to manage tickets for public transportation. In order to purchase tickets, SIMpliTravel recalls the SIMpliPay Servlet. Moreover, in order to validate them, it enables an NFC communication with an ad hoc programmed reader.

All the Servlets are loaded in the SIM card and access to the SCWS contents (html pages, images and scripts) loaded in the SIM card too.

\section{The User-Centered Design Approach}

In order to build a fully usable service portfolio and to minimize the learning curve, we have actively involved users in the development process, following the principles of the User-Centered Design approach [19]. The philosophy behind such approach is that the product should suit the user, rather than making the user suit the product [20].

\subsection{Specification of the Context of Use, Personas and Scenarios}

The first activity we performed was the specification of the context of use, which involves the description of target users, the goals they are supposed to achieve and the environment they will operate in. The primary users of SIMpliLife are tourists and in general people who frequently travel, mainly using public transportation. They have enough free time to spend in visiting touristic points-of-interest in the major European cities. Moreover, young people, aged between 18 and 35, are more likely to use SIMpliLife rather than elderly people, as they are more inclined to use a payment system via mobile phone and in general to test and use the latest mobile technologies such as NFC. They are the so-called "first adopters".

SIMpliLife is conceived to allow target users to achieve a set of goals, from information retrieval, to payment and ticketing operations. The environment in which the users will operate is variegated and characterized by mobility.

Once we defined the context of use, we were able to build more specific user's profiles, the so-called "personas" [21]. Having potential users on which you can base design assumptions is very important in order to make consistent choices in the development phase. Since SIMpliLife is a brand new system and we don't have previous users to be consulted, we adopted a set of archetypes of people who could potentially be involved in the system: The aim is that these archetypes provide us with the perception that we are creating a system for specific people, not just for generic and abstract “users". 
Another tool used for identifying users' needs and requirements is the creation of usage scenarios, which can be defined as informal narrative descriptions of how a hypothetical interaction between the users and the application we are developing might work. They are a sort of "narrative prototypes" interpreted by the abovementioned personas. The scenario technique provides a fast and effective way to imagine the use of features designed, or to create new ones.

Scenario A. Alice is a Ph.D. student, she travels very often for conferences related to her research activities. She uses Internet daily, both for work and for entertainment, and sometimes she connects to the Web using her mobile phone. Usually Alice plans carefully her travels: She collects maps and directions before leaving. This time, as her plane was late, she arrives in the city centre when it's already evening and it seems that busses are not on service anymore. She notices a SIMpliCity poster just near the bus stop and, as she already has SIMpliLife application on her NFC mobile phone (she has already travelled to other "SIMpli" cities), she immediately draws up her phone near the tag closest to her hotel: the system confirms that the last bus left an hour ago. The only solution is to call a taxi, so Alice puts her phone near the taxi tag and automatically sends an SMS to the taxi service center. Five minutes later, as announced by the feedback message Alice received by the system, a taxi arrives to pick her up and take her to the hotel. As she has no cash money to pay for the taxi, she selects "NFC Payment" on the main menu of SIMpliPay, taps her mobile phone near the NFC reader placed inside the car and pays the bill.

Scenario B. Rita is a middle-aged woman interested in history and art. She occasionally uses Internet for work. She has a mobile phone that she only uses for making phone calls and writing SMS. She has gone for a walk in the city-center and she would like to know how to reach the Pantheon from Termini train station. She notices a SIMpliCity poster and remembers that her daughter has installed something named SIMpliCity on her mobile phone just a few days ago.
She wakes up the application and touches the Pantheon tag on the Poster. In a few seconds she's redirected to the Roman public transport company website and she obtains the route on her mobile phone. As she's pleasantly surprised of the ease-of-use of the service, she decides to buy the bus ticket directly through the phone: She selects the "buy bus ticket" option and the application asks her to choose between NFC and on-line payment. As she doesn't know the difference between them, she asks for additional information: She chooses NFC payment, selects her ticket, draws up the phone to the reader placed near the bus stop and pays the due amount.

\subsection{Prototyping and Usability Test}

As the iteration of design solutions is another central point in the UCD approach, after defining these usage scenarios we built a paper-based prototype to be tested with real users. A paper-based prototype requires a small amount of time and money to be developed and is particularly useful for testing multiple design concepts as it allows to make changes at a very little cost.

For the preliminary test we involved twelve users, aged between 18 and 35 with heterogeneous education (four of them had a Master's degree, five had a Bachelor's degree and three were graduated) and an heterogeneous technological equipment: While all of the users involved have a mobile phone and use it to make phone calls and send SMS, just three of them actually used it to perform more complex actions such as managing e-mails and connecting to the Web. We asked them to go through five different tasks, selected from a list of ten; some were "simple" tasks, involving only one application (for example "buy a daily ticket for the bus"), while other were "complex" tasks (for example "finding out the opening hours of the Colosseum and buy two entrance tickets"), which involved the use of at least two different applications. When asked for an overall evaluation, all the users involved answered that they found SIMpliLife easy to 
use. Ten of them would really like to have the application installed on the mobile phone. One user was worried about the transaction fees applied to proximity and on line payment and declared to be inclined to use SIMpliLife only if there were no additional costs. For another user the bottleneck was represented by the connection costs for the access to on line services.

The remote payment was the most critical action: while three users completely trusted the PayPal system (two of them had already used it for remote payment and one had only heard about it), four of them asked for additional information about it. They would like to have a "more information" button when selecting "on line payment”. Conversely, after a short introduction about using NFC for proximity payments, nobody had problems in using such system; four users declare to appreciate the possibility of performing proximity payments with a mobile phone with no connectivity costs, in the same way that they currently use their payment card. They also appreciate the opportunity of having control over the account balance by choosing the "control credit" button: one of the users suggested keeping the current balance always visible when accessing SIMpliPay, instead of accessing it through a specific button.

Through the users' suggestions, we built a high-fidelity prototype made with Adobe ${ }^{\circledR}$ Flash ${ }^{\circledR}$ software (see Fig. 3), which is more interactive than the paper-based one and gives the user the real look-and-feel of the final application.

The other five users that haven't tested the application before found SIMpliLife easy to use and, after a short introduction about NFC technology, they had no particular problems in interacting with it. They enjoyed the proximity payment feature and trusted it more than the remote payment.

They appreciated also the "modularity" of the application, e.g., the fact that they can use SIMpliLife for performing different actions not necessarily linked between them (as an example of this, one user declared

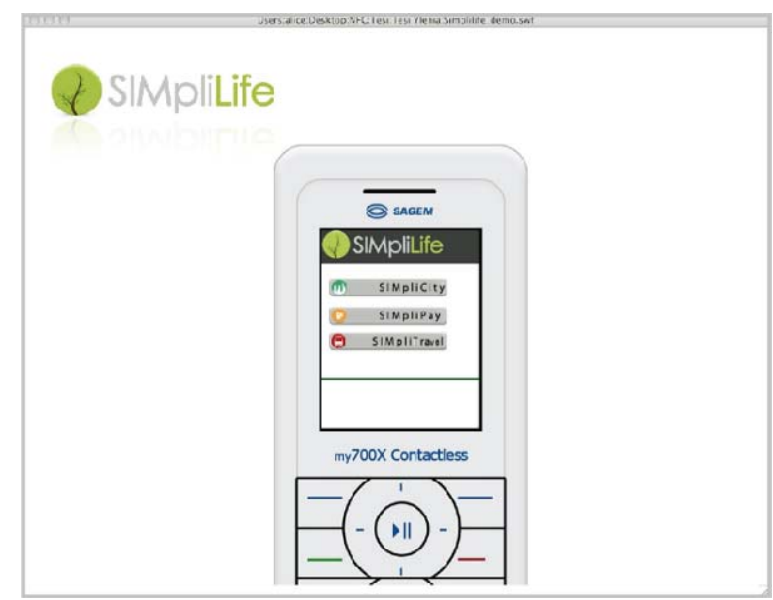

Fig. 3 The high-fidelity prototype made with Adobe ${ }^{\circledR}$ Flash ${ }^{\circledR}$ software.

to appreciate the information retrieval via SIMpliCity, but she declared also to not completely trust the payment feature, so she would use SIMpliLife just for obtaining information via RFID tags).

\section{Conclusions}

In this paper we presented SIMpliLife, a platform for NFC mobile phones that allows users to retrieve information via Smart Posters provided with RFID passive tags, to perform both remote and proximity payments and to manage tickets for public transportation. SIMpliLife was developed for a mobile phone with the SE on the SIM card: This guaranteed the portability of the application on every enabled device. Moreover, we have built SIMpliLife making use of SCWS technology, thus providing a nice, web-like user interface.

In designing SIMpliLife we followed a User-Centered Design approach, involving target users since the beginning of the design process. We built both a low-fidelity (paper-based) prototype and tested it with twelve users, and a high-fidelity prototype and tested it with ten users. The main findings of the usability tests concern the ease-of-use of the platform and the high level of satisfaction that the users experience, especially when they perform a proximity payment. Conversely, the remote payment was perceived as the most complex action: One out of 
three users asked for additional information when they had to perform a remote payment.

\section{Future Work}

We have developed a first prototype of SIMpliLife using the Sagem my700x mobile phone, a SIM card with Single Wire Protocol (SWP) and SCWS features provided by Gemalto and the Gemalto Developer Suite. As the SIM card we used is not registered on any GSM network, we have only been able to test the features that don't require an OTA connection (tag reading, NFC communication with external readers and interaction with html pages loaded into the SCWS). The external communication that requires an OTA connection (e.g., external html pages, communication via SMS) has been tested only on the emulator. The SIMpliLife suite will be soon ported on a registered SIM card; moreover we will also make it run on the newest smart phone provided with NFC technology, the Samsung (Google) Nexus S. Furthermore, we will conduct additional usability tests, providing OTA features, in order to reach the final release of SIMpliLife.

\section{References}

[1] M. Weiser, The computer for the 21st century, Scientific American 256 (1991) 94-104.

[2] M. Weiser, Some Computer Science Issues in Ubiquitous Computing, Communication of the ACM 36 (1993) 74-84.

[3] International Telecommunication Union (ITU), World Telecommunication/ICT Indicators Database 2009, 13th ed., available online at: http://www.itu.int/ITU-D/ict/publ ications/world/world.html

[4] U. Ceipidor, C. Medaglia, A. Moroni, S. Sposato, Design, prototyping and evaluation of SIMpliCity, an NFC system for the mobility of citizens, in: CHI 2009, Boston, pp. 117-120.

[5] S. Levialdi, C. Medaglia, A. Moroni, S. Sposato, NFCTicketing: a prototyping and usability test of an
NFC-based virtual ticketing application, in: First International Workshop on Near Field Communication, Hagenberg, Austria, 2009, pp. 45-50.

[6] ISO/IEC 18092:2004, Information TechnologyTelecommunications and Information Exchange between Systems-Near Field Communication Interface and Protocol (NFCIP-1), Geneva, 2004.

[7] ISO/IEC 21481:2005, Information TechnologyTelecommunications and Information Exchange between Systems-Near Field Communication Interface and Protocol -2 (NFCIP-2), Geneva, 2005.

[8] M. Reveilhac, M. Pasquet, Promising secure element alternatives for NFC technology, in: First International Workshop on Near Field Communication, 2009, pp. 75-80.

[9] Alternatives for banks to offer secure mobile payments, Mobey Forum, Version 1.0, 2010.

[10] ETSI TS 102622 V.7.5.0, Smart Cards; UICCContactless Front-End (CLF) Interface; Host Controller Interface (HCI), Release 7, 2009.

[11] ETSI TS 102613 V.7.7.0, Smart Cards: UICCContactless Front-End (CLF) Interface, part 1: physical and data link layer characteristics, Release 7, 2009.

[12] Global Platform, Card Specification Version 2.2, 2006, available online at: http://www.globalplatform.org.

[13] Available online at: http://www.gemalto.com/techno/stk/.

[14] G. Madlmayr, D. Brandlberger, J. Langer, J. Scharinger, Evaluation of smartcard webserver as an application platform from a user's perspective, in: MoMM, 2008.

[15] G. Madlmayr, J. Langer, J. Scharinger, Managing an NFC ecosystem, in: 7th International Conference on Mobile Business, 2008, pp. 95-101.

[16] GSMA: Mobile NFC Technical Guidelines, Version 2.0, 2007.

[17] GSMA: Mobile NFC Services, Version 1.0, 2007.

[18] GSMA: Pay-Buy-Mobile Business Opportunity Analysis —Public White Paper, Version 1.0, 2007.

[19] ISO/IEC 13407:1999(E), Human-Centred Design Processes for Interactive Systems, Geneva, 1999.

[20] C. Courage, K. Baxter, Understanding Your Users: a Practical Guide to User Requirements Methods, Tools, and Techniques, Morgan Kaufmann Publisher, 2005.

[21] A. Cooper, The Inmates Are Running the Asylum: Why High Tech Products Drive Us Crazy and How to Restore the Sanity, Sams Publishing, 1999. 